\title{
EVALUACIÓN DE LAS MEDIDAS DE ATENCIÓN A LA DIVERSIDAD EN LA EDUCACIÓN PRIMARIA EN GALICIA: IMPACTO ESCOLAR
}

\author{
EVALUATION OF THE MEASURES OF ATTENTION TO THE DIVERSITY \\ IN THE PRIMARY EDUCATION IN GALICIA: SCHOOL IMPACT
}

\author{
José Domínguez Alonso* \\ Centro de Educación Infantil y Primaria \\ Margarita R. Pino Juste** \\ Universidad de Vigo
}

\section{RESUMEN}

El presente estudio tuvo como finalidad analizar el uso de las diferentes medidas de atención a la diversidad del alumnado en la Comunidad Autónoma Gallega a través de una escala diseñada para el efecto. Los resultados obtenidos indican una elevada presencia de las medidas ordinarias frente a las extraordinarias. Además dentro de las medidas ordinarias se manifiesta un predominio del refuerzo educativo (RE). También reseñar, que no se encontraron diferencias estadísticamente significativas de las medidas de atención a la diversidad con respecto a las variables independientes (género, edad, experiencia, titulación y sistema de acceso), obteniendo una elevada consistencia interna en la escala elaborada (EMAD).

Palabras clave: Atención a la diversidad, escuela para todos, orientación escolar.

\section{ABSTRACT}

The aim this study is to analyze the use of different approaches to address students diversity in the Autonomous Region of Galicia, through a scale designed for that effect. The

\footnotetext{
* José Domínguez Alonso es Doctor en Psicopedagogía por la Universidad de Vigo. Las investigaciones realizadas se orientan hacia el estudio de la atención a la diversidad en la enseñanza obligatoria, en cuyo campo cuenta con numerosos artículos publicados. E-mail: jdalonso@edu.xunta.es

** Margarita R. Pino Juste. Doctora en Pedagogía por la Universidad de Santiago de Compostela. Es Profesora titular del Departamento de Didáctica, Organización Escolar y Métodos de Investigación en la Universidad de Vigo. Sus líneas de investigación están centradas en diversidad y resolución de conflictos en el aula, educación para la salud e intervención educativa con colectivos en riesgo social. E-mail: mpni@uvigo.es
} 
main purpose of this scale is to evaluate current approaches in the educational system, especially those in the of the primary education level.

Results indicate that, ordinary approaches are present more often that extraordinary approaches. Furthermore, there is a predominance of the educational reinforcement (RE). It is also outlined that there were not statistically significant differences between different approaches regarding the independent variables (sex, age, experience, qualifications and access routes), obtaining a high internal consistency in the elaborated scale (EMAD). This article justifies the need of a constant review of the existing needs in educational canters to be able to establish educational and formative coherent answers for the diversity of students. Consequently, the final model of integration-inclusion school must be the response to the diversity from ordinary education, giving a common offer to all students and at the same time differentiated answers for each one of them, adjusted to their individual characteristics.

Key words: Diversity, Inclusion, education for all, school counselling, guidance.

"Nuestros sistemas educativos afrontan, por primera vez en la historia, una nueva etapa absolutamente revolucionaria: intentar acabar con la pedagogía de la exclusión"

(TOMLINSON, 2001: 9)

\section{Introducción}

A día de hoy, la diversidad es un principio inherente a cualquier realidad social que está presente en todos los centros educativos (universitarios y no universitarios; públicos, concertados y privados) manifestándose de diferentes modos y en distintos grados sobre todos los elementos que conforman la comunidad educativa, pero sobre todo en la doble predisposición existente en los colectivos que se encargan de atender al alumnado diverso en sus centros:

Por un lado, los que consideran la diversidad como una gran oportunidad educativa de carácter individual, escolar y social que va a beneficiar a todo el alumnado.

Por otro, los que consideran su presencia como un estorbo que impiden el funcionamiento normal de la clase, alcanzar niveles más elevados de eficacia y sobre todo que afectan al rendimiento global del sistema. Destacar aquí, con Essomba (2003), que se aprecia un predominio de los discursos favorables (primera opción) pero con un sentimiento poco real en la práctica educativa, infiriendo que la atención a la diversidad es uno de los argumentos más manidos en el ámbito educativo que se utiliza reiteradamente como recurso para atender y englobar a todo el alumnado, pero no se concreta en medidas que corrijan las deficiencias o lagunas existentes en este campo que contribuyan de manera efectiva a favor de este colectivo que tiene los mismos derechos que el resto del alumnado. Así, la atención a la diversidad termina convirtiéndose en un reto difícil, complicado y a veces contradictorio. 
A pesar de ello, se tiene el convencimiento, que el referente final de la integración-inclusión escolar debe ser la respuesta a la diversidad desde la educación ordinaria, ofreciendo una oferta común para todo el alumnado y al mismo tiempo repuestas diferenciadas para cada uno de ellos y ajustadas a sus características individuales. En esta línea, Aguilar (2000: 104) sostiene que "si logramos situaciones eficaces de aprendizaje para los alumnos con necesidades educativas especiales dentro de nuestras instituciones educativas ordinarias, estaremos forjando, a la vez, el mejor contexto educativo para todo el alumnado" para ello será preciso diseñar estrategias muy variadas (Sobrado Fernández, 2006).

Estos principios demuestran la importancia de evaluar las distintas medidas de atención a la diversidad con el fin de desarrollar posibles vías de actuación que se adecuen más a la necesidades educativas. La finalidad del estudio reside en diseñar una escala de valoración de las medidas de atención a la diversidad en la educación primaria en Galicia, con el doble objetivo de identificar, por un lado la importancia que tienen dichas medidas para el profesorado y, por otro, su impacto escolar en los centros educativos.

\section{Marco teórico-contextual}

El estudio de las desigualdades sociales en educación iniciado en los años cincuenta en Inglaterra, se convierte en una línea de trabajo importante durante esta época y la siguiente. Su procupación fundamental (De Pablo, 1984) es la de hacer ver la situación de desventaja e injusticia en la que se encuentran los sectores más bajos de la sociedad, incapaces de desenvolver todo su potencial humano debido al desigual acceso que tienen a los distintos niveles del sistema educativo. Se confía en la capacidad de la educación para producir cambios sociales, considerando la educación compensatoria como una importante estrategia de política educativa.

No obstante, esta línea de trabajo se modifica en la década de los sesenta, debido sobre todo a la publicación en el año 1966 del Informe Coleman, el cual indicaba que la igualdad de oportunidades en la educación implicaba no solamente escuelas iguales, sino también escuelas de igual eficacia donde su influencia supere las diferencias iniciales de los niños/as que provienen de grupos sociales diferentes. Se empezaba a ver el fracaso de la escuela como instrumento de cambio y de transformación social, llegando a afirmar que el sistema educativo contribuía a reproducir el orden social y que no es función suya la transformación de la sociedad. Aún así, sigue existiendo una linea de investigación encaminada a demostrar que la institución escolar ejerce influencia en el aprendizaje de los alumnos, sea cual sea su procedencia social.

Los diferentes trabajos llevados a cabo en esta época en el ámbito de la educación especial ponen de relieve el estudio de la atención a la diversidad desde un tratamiento contextualizado (García Pastor, 1993; Wang, 1995), diferenciando el contexto donde se perciben las intervenciones educativas ( necesidades educativas especiales como hecho innovador) del que emanan las decisiones para satisfacer las necesidades. Otro de los ámbitos educativos investigados es el de la educación compensatoria, que se ocupa del alumnado que experimenta dificultades en su desarrollo escolar asociadas a un determinado origen socioeconómico. En este campo (CIDE, 1998), se encuentran los análisis de situaciones (estudios sociológicos e interdisciplinares), programas, profesorado, centros y métodos de intervención (modificación cognitiva, capacidad psicolingüística, programas generales y tecnología). 
En síntesis, decir que las investigaciónes en el ámbito de la educación especial anteriores a la década de los noventa, se centran en los distintos tipos de discapacidades (visuales, motóricas, auditivas, psíquicas, intelectuales y del lenguaje, entre otras).

A partir de 1990, con el desarrollo de la LOGSE, la mayoría de las investigaciones realizadas hacen referencia a la integración del alumnado con necesidades educativas especiales tanto en la escuela como en el mundo laboral. La temática de la integración cobra especial interés a partir de su generalización en el sistema educativo, implicando un cambio en la tendencia de los estudios sobre la atención a la diversidad del alumnado que hasta el momento estaban centrados en las diferentes discapacidades. Desde este horizonte, la incorporación paulatina del alumnado con necesidades educativas especiales a los centros ordinarios, se establece como un reto del propio sistema educativo y como uno de los problemas más importantes a resolver por la dificultad que entraña "proporcionar una respuesta educativa adecuada a un colectivo de estudiantes ciertamente heterogéneo, con necesidades de formación muy diversas e intereses personales diferentes, operando en un mismo centro escolar y con un currículo en gran parte común” (MEC, 1989: 119).

Con el paso del tiempo, los enfoques sobre la atención al alumnado diverso abandonan los planteamientos centrados en el déficit y se van acercando a los competenciales (López Melero, 1997) o culturales/integradores (García Pastor, 1993) de la respuesta educativa a las necesidades del sujeto en un contexto determinado. Según Parrilla (2004), el nuevo sentido de la investigación en la atención a la diversidad debe encaminarse a la configuración de un cuerpo de trabajo más abierto a la diversidad, más constructivo, vinculado al contexto, más atento a la relevancia y significación socio-educativa, que permita el desarrollo de nuevas teorias e ideas relevantes no solamente para el conocimiento sino también para la mejora de la calidad educativa.

Sin embargo, la atención a la diversidad de todo el alumnado y no solamente de los que presentan dificultades de aprendizaje y los procesos de cambio que esto conlleva, sigue cuestionando los supuestos que encubren las teorías y prácticas educativas. La preocupación para la investigación en este nuevo campo es conocer como producir ese cambio, como hacerlo posible y como desarrollar estrategias válidas para reestructurar las escuelas de forma que puedan responder a las necesidades de todo el alumnado. Estudios e investigaciones en esta perspectiva, señalan como condición básica genérica para el desarrollo de una escuela adaptada a la diversidad la mejora y transformación de la escuela tradicional, destacando el papel que juega el desarrollo de procesos de apoyo en las instituciones escolares. En esta línea están los trabajos de Hopkins, Ainscow \& West, 1994; Ainscow, 1995; Lypsky \& Gartner, 1996; Ainscow, Ferrel \& Tweddle, 1998; Ballard \& MacDonald, 1998; Clark, Dyson, Millward \& Robson, 1999.

En este punto, Gallego Vega (2002) señala que, las investigaciones llevadas a cabo sobre la construcción de marcos de apoyo a la diversidad en el ámbito anglosajón (Daniels \& Norwich, 1992; Hart, 1992), y en contextos más cercanos al nuestro (Gallego \& Hernández, 1999), concluyen en la necesidad de abandonar la concepción más tradicional y extendida del apoyo y asesoramiento (como apoyo terapéutico y asesoramiento clínico centrados ambos en el alumnado) y avanzar hacia la concepción de un apoyo que parta de premisas bien distintas sobre la diversidad, la escuela y los profesionales implicados en la misma.

Sin embargo, no debe olvidarse que el ámbito teórico y el práctico no son dos aspectos aislados e independientes, sino que se influyen mutuamente, y las decisiones que se adopten 
en uno de los ámbitos afectará al otro, "el profesorado debe reflexionar sobre las intenciones que han de guiar y justificar su práctica y, al mismo tiempo, debe analizar y reflexionar sobre la coherencia del plan de acción con el que se pretende enseñar y con el tipo de aprendizaje que se pretende conseguir" (Xunta de Galicia, 1993: 21).

En este estudio nuestra pretensión es evaluar las medidas de atención a la diversidad que existen en la actualidad en el sistema educativo español, en lo que se refiere a su presencia en la etapa de la educación primaria en la Comunidad Autónoma de Galicia.

\section{Método}

Desde una perspectiva indagadora y con la necesidad de lograr una mejor aproximación a las prácticas docentes en los centros educativos, hemos lleva a cabo una investigación cuasi-experimental, en la que el "investigador no ejerce una influencia directa sobre el problema de investigación, lo que significa que no controla ni manipula los factores o fenómenos que influyen en el comportamiento o rendimiento de los sujetos", de tipo descriptivo, "simplemente proporcionan información sobre opiniones, hechos o fenómenos" (Cardona, 2002: 36-37). La única pretensión de la misma, será la identificación desde el punto de vista de los orientadores de la situación que prevalece en los centros de primaria en lo referente a las medidas de atención a la diversidad, desde las percepciones, actitudes, opiniones, preferencias, características o prácticas educativas que se llevan a cabo en los mismos.

\section{Participantes}

Para la verificación de este estudio se ha utilizado una muestra de orientadores con edades comprendidas entre los 30 y 60 años, con unos porcentajes del 44,5\% (tramo de 41 a 50 años) y un $24,5 \%$ (tramo de 30 a 40 años). Además se ha tenido presente el género (Varones $=22,7 \%$, Mujeres $=66,4 \%$ ); la experiencia educativa (entre 11 e 18 anos $=24,7 \%$, más de 18 años $=53,6 \%$ ); y formación (Diplomados $=99,3 \%$, Licenciados $=64,8 \%$ ). Todos los sujetos pertenecían a centros públicos de educación infantil y primaria (CEIP) con características socioeconómicas similares, quedando compuesta la muestra total por 140 participantes que representan el 61,14\% de la población total (sobre una población de 229).

\section{Instrumento}

La escala de valoración utilizada en nuestro estudio ha sido de 9 ítems que se corresponden con las distintas medidas de atención a la diversidad que existen en el sistema educativo (centros de educación primaria). En las respuestas a cada pregunta se utiliza la escala de Likert formada por cinco categorías (0-1-2-3-4), diseñada de forma que se evita al máximo los fenómenos de contaminación y perseveración en las respuestas. El objetivo esencial de esta escala es evaluar las medidas de atención a la diversidad que existen en la actualidad en el sistema educativo español, especialmente en lo que se refiere a la presencia de estas medidas en la etapa de la educación primaria (Pino, Domínguez \& López-Castedo, 2007). El coeficiente Alfa de Cronbach para el total de la muestra fue de .91, y en el método de las dos mitades se obtiene una correlación de .89 en la primera parte y .79 en la segunda. 


\section{Procedimiento}

La estrategia básica de aplicación en esta investigación consistió en administrar la escala de valoración a través del correo electrónico en un archivo adjunto, carta de presentación e instrucciones pertinentes para cubrirlo, y con un tiempo de respuesta de 20 días. La mortalidad experimental fue del $38,86 \%$. La duración total de respuesta de la prueba fue, aproximadamente de 10 minutos.

\section{Resultados}

Para interpretar los resultados se han agrupado las cinco modalidades de respuesta de la escala (nada, poco, suficiente, bastante, mucho) en tres (nada/poco, suficiente, bastante/mucho). En el estudio se llevan a cabo tres tipos de análisis:

\section{Análisis descriptivo}

Las medidas de atención a la diversidad que existen en los centros educativos actualmente son consideradas como buenas $(46 \%)$, frente a un $27 \%$ que las considera regulares y un $12 \%$ muy flojas. En el total de la muestra, las medidas ordinarias mejor valoradas han sido la acción tutorial y orientadora $(67 \%)$ - posiblemente debido a ser orientadores los que respondieron la escala-, y el refuerzo educativo (61\%). Las menos apreciadas son los agrupamientos flexibles (44\%) y la atención a las minorías étnicas $(38 \%)$. Por otro lado, de las medidas extraordinarias, la más frecuente es la adaptación curricular individualizada - ACI - (38\%) y la menos, la permanencia o reducción de un año más en el ciclo $(32 \%)$ (Tabla 1).

Como síntesis de las valoraciones aportadas sobre las medidas de atención a la diversidad, destacar la diferencia existente entre los porcentajes de valoración de las medidas ordinarias $(56 \%)$ frente a las extraordinarias (38\%). Esto nos lleva a considerar que las medidas ordinarias son las mejor valoradas y más utilizadas en los centros de educación primaria en Galicia.

\section{Análisis inferencial}

El análisis inferencial se llevó a cabo bajo dos situaciones, pero siempre con el objetivo de determinar con precisión la probabilidad de significación en cada variable (McMillan \& Schumacher, 2005). En primer lugar (para la variable sexo), se utiliza un diseño unifactorial intersujetos - diseño de dos grupos aleatorios - (prueba t, media, desviación típica y las correspondientes a la prueba de muestras independientes), fijando la significación bilateral en $p$ menor o igual a .05. En segundo lugar (para las variables: edad, experiencia docente, experiencia en un departamento de orientación, titulaciones académicas y sistema de acceso), se emplea un diseño unifactorial intersujetos — multigrupo-, utilizando la análisis de varianza (anova) de un factor.

Se comprueba si existen diferencias significativas entre las puntuaciones de las medidas ordinarias y extraordinarias con respecto al sexo, confirmando que no existen diferencias significativas. A continuación se comprueba si existen diferencias estadísticamente signifi- 
TABLA 1: Medidas ordinarias versus medidas extraordinarias.

\begin{tabular}{|c|c|c|c|c|}
\hline \multirow{2}{*}{$\begin{array}{l}\text { Presencia de las medidas ordinarias/ } \\
\text { extraordinarias }\end{array}$} & \multicolumn{4}{|c|}{ Porcentajes } \\
\hline & Nada/poco & Suficiente & $\begin{array}{l}\text { Bast.I } \\
\text { Mucho }\end{array}$ & $\begin{array}{l}\mathrm{Nc} / \\
\mathrm{ns}\end{array}$ \\
\hline En los documentos del centro & $15 \%$ & $29 \%$ & $44 \%$ & $12 \%$ \\
\hline En el aula & $14 \%$ & $23 \%$ & $50 \%$ & $13 \%$ \\
\hline En la acción tutorial y orientadora & $5 \%$ & $21 \%$ & $67 \%$ & $7 \%$ \\
\hline Refuerzo educativo (RE) & $4 \%$ & $27 \%$ & $61 \%$ & $8 \%$ \\
\hline Agrupamientos flexibles & $15 \%$ & $18 \%$ & $44 \%$ & $21 \%$ \\
\hline Minorías étnicas & $14 \%$ & $23 \%$ & $38 \%$ & $26 \%$ \\
\hline \multicolumn{5}{|c|}{ Porcentaje medio total (M. Ordinarias): $\mathbf{5 6 \%}$} \\
\hline Permanencia o reducción de un año más en el ciclo & $18 \%$ & $36 \%$ & $32 \%$ & $14 \%$ \\
\hline Adaptaciones Curriculares Individuales (ACI) & $13 \%$ & $39 \%$ & $38 \%$ & $10 \%$ \\
\hline Acciones de atención a la diversidad cultural & $10 \%$ & $25 \%$ & $36 \%$ & $29 \%$ \\
\hline
\end{tabular}

Porcentaje medio total (M. Extraordinarias): $\mathbf{3 8 \%}$

Fuente: elaboración propia.

cativas entre las medias de las medidas ordinarias y extraordinarias en relación con los grupos de edad, experiencia docente, experiencia en departamentos de orientación, titulaciones académicas y sistema de acceso. Aquí, el estadístico de Levene revela que los grupos tienen varianzas homogéneas en todos los casos, y el análisis de la varianza muestra que no son fuentes significativas de variación (Tabla 2).

\section{Análisis factorial}

En tercer lugar, calculamos la validez de constructo del cuestionario realizando un análisis factorial a través del método de los componentes principales: cálculo de la matriz capaz de expresar la variabilidad conjunta de todas las variables, la extracción del número óptimo de factores, la rotación de la solución para facilitar su interpretación y la estimación de las puntuaciones de los sujetos en las nuevas dimensiones. Previamente se comprueba con la medida de adecuación muestral de Kaiser-Meyer-Olkin, si la muestra es aceptable para la factorización de las variables, obteniendo un índice $\mathrm{KMO}=.883$ (muy próximo a .8 , que es lo normalmente exigible), a la vez que la matriz de correlaciones anteriormente exhibida es esférica $(p<.01)$ y en consecuencia analizable desde el punto de vista factorial. La prueba de esfericidad de Bartlett, establece que la matriz puede ser factorizada, con un $X^{2}=397.074$ y una probabilidad asociada de $p=.000$, lo cual nos indica una alta significación y la aceptación de la factorización de la matriz.

A continuación se analiza la varianza total para saber los porcentajes acumulados de los factores (método de extracción: análisis de componentes principales) y procedemos a la rotación de la misma (matriz de componentes rotados), método por el cual se reparte la varianza de los factores significativos con el objeto de facilitar la interpretación de las variables abstractas resultantes (Tabla 3 ). 
TABLA 2: Medias, desviaciones típicas y prueba $t$ en función del género. Análisis de varianza (ANOVA) en función de la edad, experiencia docente, experiencia en departamentos de orientación, titulación académica y sistema de acceso.

\begin{tabular}{|c|c|c|c|c|c|}
\hline Medidas & Variables & \multicolumn{4}{|c|}{ Estadísticos } \\
\hline \multirow{6}{*}{ Ordinarias } & Sexo & $\begin{array}{l}q X=19.42 \\
\partial^{\prime} X=19,33\end{array}$ & $\begin{array}{l}S=3.91 \\
S=3.63\end{array}$ & $\begin{array}{l}t=.093 \\
t=.093\end{array}$ & $\begin{array}{l}p=.904 \\
p=.904\end{array}$ \\
\hline & Edad & & $F=1.255$ & $p=.295$ & \\
\hline & Experiencia docente & & $F=.433$ & $p=.824$ & \\
\hline & Experiencia D.O. & & $F=.697$ & $p=.557$ & \\
\hline & Titulación académica & & $\mathrm{F}=.013$ & $p=.987$ & \\
\hline & Sistema de acceso & & $F=.609$ & $p=.658$ & \\
\hline \multirow{6}{*}{ Extraordinarias } & Sexo & $\begin{array}{l}+X=10.57 \\
\delta X=10,43\end{array}$ & $\begin{array}{l}S=2.51 \\
S=2.21\end{array}$ & $\begin{array}{l}t=.187 \\
t=.187\end{array}$ & $\begin{array}{l}p=.853 \\
p=.853\end{array}$ \\
\hline & Edad & & $F=.605$ & $p=.661$ & \\
\hline & Experiencia docente & & $F=1.041$ & $p=.401$ & \\
\hline & Experiencia D.O. & & $F=.297$ & $p=.827$ & \\
\hline & Titulación académica & & $F=.253$ & $p=.778$ & \\
\hline & Sistema de acceso & & $F=.547$ & $p=.702$ & \\
\hline
\end{tabular}

Fuente: elaboración propia.

TABLA 3: Varianza total y rotación factorial de las medidas de atención a la diversidad.

\begin{tabular}{|l|c|c|}
\hline \multicolumn{1}{|c|}{ Ítem } & Factor $\mathbf{1}$ & Factor $\mathbf{2}$ \\
\hline 3. Acción tutorial e orientadora & .879 & .873 \\
2. Medidas ordinarias a nivel de aula & .785 \\
4. Refuerzo Educativo (RE) o Adaptaciónes curriculares no significativas & .770 & .741 \\
6. Medidas ordinarias de atención a las minorías étnicas & .711 & \\
5. Medidas organizativas & & .810 \\
1. Medidas ordinarias a nivel de centro & .793 \\
8. Adaptaciones curriculares significativas (ACI) & .678 \\
9. Medidas extraordinarias de atención a la diversidad cultural & \\
7. Permanencia o reducción de un año más en el ciclo & 5.39 & 1.03 \\
\hline VARIANZA & 59.9 & 11.4 \\
\hline \% VARIANZA & & .9 \\
\hline
\end{tabular}

Fuente: elaboración propia. 
El análisis factorial de los ítems de la escala fue realizado para la selección de variables de cada factor, recogiendo solamente las saturaciones superiores a .40. Así, el análisis de componentes principales nos muestra una escala bifactorial con autovalores que excedieron de la unidad y arrojan una variación total de $71,37 \%$. Se observa que la mayor varianza extraída recae sobre el primer factor con un autovalor de 5.39 siendo considerado el Factor Principal. El segundo factor que emerge en este análisis presenta un autovalor de 1.03. Con relación a los resultados obtenidos se pone de manifiesto que en el primer factor se agrupan 6 ítems: 3, 2, 4, 6, 5, y 1, que hacen referencia a las medidas de carácter ordinario "Acción tutorial y orientadora", "Medidas ordinarias a nivel de aula", "Refuerzo Educativo (RE) o Adaptaciónes curriculares no significativas", "Medidas ordinarias de atención a las minorías étnicas", "Medidas organizativas", "Medidas ordinarias a nivel de centro". Se ha denominado a este factor como MEDIDAS ORDINARIAS. Como podemos comprobar se relacionan con las más sencillas de trabajar y aplicar en los centros. En cuanto al segundo factor, se concentran 3 ítems: 8, 9 y 7, denominando a este factor como MEDIDAS EXTRAORDINARIAS. Hacen referencia a las medidas: "Adaptaciones curriculares significativas (ACI)", "Medidas extraordinarias de atención a la diversidad cultural", "Permanencia o reducción de un año más en el ciclo". Se identifican con las medidas de atención a la diversidad más extremas, de difícil aplicación y que marcan la competencia del individuo en cuanto a su capacidad de enfrentamiento y preparación adecuada de estas medidas.

A tenor de los resultados obtenidos podemos afirmar que la consistencia interna de la escala de valoración de las medidas de la atención a la diversidad creada (EMAD), en esta muestra de orientadores españoles, es muy satisfactoria con una tendencia bastante uniforme en cuanto a las variables estudiadas.

\section{Conclusiones y propuestas de mejora}

Actualmente existe en las comunidades educativas una preocupación prioritaria sobre la atención de la diversidad en los centros (Aguado-Odina, 2000), y en particular sobre las medidas o propuestas que se están llevando a cabo en el tratamiento de las diferencias educativas (Navas \& Torregrosa, 2004). Del estudio realizado se comentan algunas aportaciones.

Del análisis descriptivo se observa, a nivel general, que las medidas de atención a la diversidad consideradas ordinarias son más valoradas y utilizadas $(61 \%)$ que las extraordinarias $(39 \%)$. Aquí, dentro de las medidas ordinarias las más utilizadas son el refuerzo educativo (61\%) y la acción tutorial y orientadora (67\%), mientras que en las consideradas como extraordinarias son las adaptaciones curriculares individuales $(38 \%)$ y la atención a la diversidad cultural $(36 \%)$.

A nivel inferencial, no se aprecian diferencias estadisticamente significativas en las medidas de atención a la diversidad en función del género, edad, experiencia docente, experiencia en los departamentos de orientación, titulaciones y sistema de acceso a la orientación de los orientadores que participaron en el estudio.

En el análisis factorial se obtiene una escala bifactorial al agrupar las medidas en dos factores (explican el $71,37 \%$ de la varianza de los datos); el primer factor se corresponde con las medidas consideradas ordinarias $(59,94 \%)$ y el segundo con las extraordinarias $(11,44 \%)$, lo que viene a verificar y reforzar la estructura del cuestionario utilizado. 
Las distintas investigaciones relacionadas con las medidas de la atención a la diversidad vienen a confirmar nuestros resultados. En concordancia con nuestro estudio, Alvarez et al. (2002) concluyen respecto a las medidas de atención a la diversidad, que se llevan a cabo en los centros de enseñanza secundaria, que la adaptación curricular y el refuerzo educativo son las dos medidas más practicadas y realizadas por el profesorado. También para Torres (1999: 151) es "de todas las estrategias que pueden emplearse en el tratamiento de la diversidad, el refuerzo educativo es el más común". El mismo autor señala que su uso es muy habitual, pero no siempre está sistematizado por parte de los docentes, que echan mano de él para reforzar las explicaciones de determinados conceptos o procedimientos que presentan una especial dificultad al grupo o a un alumno/a concreto.

Respecto a las propuestas de mejora:

- Debería hacerse una valoración, fundamentalmente desde los departamentos de orientación (Hernández \& Gomariz, 2003; Menéndez, 2004), de las medidas de atención a la diversidad utilizadas y su repercusión en la vida diaria de los colegios, para elaborar por parte de las administraciones educativas programas de atención al alumnado diverso y establecer líneas de actuación y orientaciones o modelos de prevención y de intervención.

- Entrenar en las nuevas medidas (enseñanza cooperativa, aulas multinivel, incorporación de las nuevas tecnológias - TIC — ...) al profesorado (García-Bracete, García-Castellas \& Doménech, 2005), puede resultar un medio útil y adecuado en los procesos de enseñanza-aprendizaje de este alumnado.

- Es importante seguir en la línea iniciada por la Xunta de Galicia en la elaboración de guías orientativas y de respuestas educativas dedicadas al alumnado diverso, y dirigidas al profesorado ordinario no especialista, a las familias y colectivos que buscan un primer acercamiento sencillo a esta temática.

Para finalizar, decir que para conseguir incrementar la calidad de la enseñanza teniendo en cuenta la atención a la diversidad del alumnado, pasa por situar, analizar o valorar las distintas medidas que se proponen para alcanzarla, se convierte en uno de los elementos claves del sistema educativo. De esta manera, las medidas de atención a la diversidad en los centros en Galicia dependen de la etapa (infantil, primaria o secundaria) y del tipo de alumnado (con necesidades educativas de apoyo educativo, con necesidades educativas temporales, y con necesidades educativas específicas asociadas a condiciones desfavorecidas). Las metas de este estudio no son otras que las de ayudar a los destinatarios a revisar su manera de pensar y actuar con relación al alumnado que experimenta dificultades en el proceso de enseñanza-aprendizaje, sean cuales sean las causas que las originaron, además de proporcionar una visión real de los centros educativos en la atención del alumnado diverso.

\section{Referencias bibliográficas}

Aguado Odina, $M^{a}$ T. (2000). "Diversidad, igualdad, cultura escolar: significado e implicaciones prácticas en la Enseñanza Secundaria Obligatoria". REOP, 11 (2), 187-198.

Aguilar Montero, L. A. (2000). De la integración a la inclusividad. La atención a la diversidad: Pilar básico en la Escuela del Siglo XXI. Buenos Aires: Espacio Editorial.

Ainscow, M. (1995). Necesidades educativas especiales. Madrid: Narcea-UNESCO. 
Ainscow, M., Farrell, P. \& Tweddle, D. (1998). "Developing policies for inclusive education: a study of the role of local education authorities". Internacional Journal of Inclusive Education, 4 (3), 211-229.

Álvarez, V.; Rodriguez, A.; García, E.; Gil, J.; López, I.; Romero, S.; Padilla, Mª T.; García, J. \& Correa, J. (2002). "La atención a la diversidad en los centros de enseñanza secundaria: estudio descriptivo en la provincia de Sevilla". Revista de Investigación Educativa, 20 (1), 225-245.

Ballard, K. \& MacDonald, T. (1998). "New Zealand: Inclusive school, inclusive philosophy?”. En T. Booth \& M. Ainscow (Eds.): The From to us, (68-94). London: Routledge.

Boza, A. (2001). "Los equipos de orientación de sector. Funciones y modelos de intervención". Revista española de orientación y psicopedagogía, 12, 50-71.

Cardona Moltó, $\mathrm{M}^{\mathrm{a}}$ C. (2002). Introducción a los Métodos de Investigación en Educación. Madrid: EOS.

CIDE (1998). Catorce años de investigación sobre desigualdades en educación en España. Madrid. Ministerio de Educación y Cultura, Centro de investigación y Documentación Educativa, Área de Estudios e Investigación. Núm. 133.

Clark, C.; Dyson, A.; Millward, A. \& Robson, S. (1999). "Inclusive education and schools as organizations". International Journal of Inclusive Education, 3 (1), 37-51.

Daniels, H. \& Norwich, B. (1992). "Support from the team". Managing Schools Today, 1 (6), 30-31.

De Pablo, A. (1984). "Causalidad, estructura y acción social: consideraciones entorno a la sociología de la educación". Revista de Educación, 224, 25-47.

Essomba Gelabert, M. A. (2003): Els discursos sobre atenció a la diversitat en la comunitat educativa a Catalunya. UAB (Tesi Doctoral).

Gallego, C. \& Hernández, E. (1999). "El apoyo a la diversidad en la escuela: experiencias y modelos innovadores". En M. A. Verdugo \& F. B. Jordán de Urríes (Coords.): Hacia una nueva concepción de la discapacidad, (563-580). Salamanca: Amarú.

Gallego Vega, C. (2002). "El apoyo entre profesores como actividad educativa inclusiva". Revista de Educación, 327, 83-105.

García Bracete, F. J.; García Castellas, R. \& Doménech Betoret, F. (2005). “Características, dificultades y necesidades formativas de los profesores tutores de educación infantil y primaria en los centros escolares de la provincia de Castellón”. REOP, 16 (2), 199-224.

García Pastor, C. (1993). Una escuela común para niños diferentes: la integración escolar. Barcelona: PPU.

Hart, S. (1992). “Collaborative classrooms". En T. Booth, W. Swan, M. Masterton \& P. Potts (Ed.): Learning for all I, curricula for diversity in education, (9-22). London and New York: The Open University.

Hernández, J. \& Gomariz, Ma A. (2003). "Los departamentos de orientación en la región de Murcia: orientación y atención a la diversidad". REOP, 14 (2), 129-147.

Hopkins, D.; Ainscow, M. \& West, M. (1994). School Improvement in an Era of Change. Londres: Cassell.

Lipsky, D. \& Gartner, A. (1996). "Inclusion, School Restructuring and the remaking of American Society". Harvard Educational Review, 66 (4), 762-795.

López Melero, M. (1997). "La educación (especial): ¿Hija de un Dios Menor en el Mundo de la Ciencia de la Educación?". Educar, 21, 7-17.

McMillan, J. H. \& Schumacher, S. (2005). Investigación Educativa. Una introducción conceptual. Madrid: Pearson Educación ( $5^{\text {a }}$ edición). 
Menéndez Martín, E. (2004). "Evolución de los servicios de orientación en la Comunidad Autónoma de Galicia: situación actual”. REOP, 15 (2), 401-411.

Ministerio de Educación y Ciencia, (1989). Adaptaciones curriculares y organización escolar. Conferencias del seminario hispano-británico. Serie Documentos. Madrid: MEC.

Navas Martínez, L. \& Torregrosa Gironés, G. (2004). “Algunas variables predictoras de las actitudes del alumnado ante la integración escolar". REOP, 19 (2), 159-171.

Parrilla Latas, A. (2004). "La atención a la diversidad en Andalucía". En G. Echeita \& M. A. Verdugo: La Declaración de Salamanca sobre Necesidades Educativas Especiales 10 años despues. Valoración y prospectiva. Salamanca: Publicaciones INICO.

Pino, M., Domínguez, J. \& López-Castedo, A. (2007). "Evaluating Appreciation of Measures Attending to Pupil Diversity (EMAD)". Psychological Reports, 100, 783-786.

Repetto Talavera, E. 2001. "Evolución histórica de la orientación educativa". Bordón: Revista de orientación pedagógica, 53 (2), 287-298.

Sobrado Fernández, L. M. \& Santos Rego, M. A. (Coords.) (2004). Orientación escolar e acción titorial en Galicia. Informe sobre o estado e a situación do sistema educativo en Galicia. Consello Escolar de Galicia. Xunta de Galicia. Santiago de Compostela.

Sobrado Fernández, L. M. (Dir.) (2006). A calidade educativa dos centros docentes. Valoración e desenvolvemento. ICE. Universidade de Santiago de Compostela.

Tomlinson, C. A. (2001). El aula diversificada: dar respuesta a las necesidades de todos los alumnos. Barcelona: Octaedro ( $1^{\mathrm{a}}$ ed.).

Torres González, J. A. (1999). Educación y diversidad. Bases didácticas y organizativas. Málaga: Aljibe.

Wang, M. (1995). Atención a la diversidad del alumnado. Madrid: Narcea.

Xunta de Galicia (1993). O proxecto curricular de centro, (5). Santiago de Compostela. Consellería de Educación e Ordenación Universitaria.

Fecha de finalización del artículo: 12-10-1997

Fecha de recepción 24-10-2007

Fecha de revisión:05-03-2009

Fecha de aceptación:05-05-2009 